\title{
Computer-controlled in-class feedback system for interactive lectures
}

\author{
T. J. Bensky \\ Physics Department, California Polytechnic State University, San Luis Obispo, San Luis Obispo, \\ California 93407
}

\begin{abstract}
Instructors who wish to implement an interactive lecture style, such as peer instruction, have the need to collect feedback from students in a lecture environment. We present a computer-controlled electronic circuit that allows for quick, rigorous, and accurate measurement and reporting of student feedback in the lecture environment.
\end{abstract}

\section{INTRODUCTION}

Peer Instruction (PI) is a method that creates a more interactive lecture environment. Lectures are suspended at regular intervals to offer students short conceptual questions. The questions are generally taken from the material just presented and are designed to address common misunderstandings among the students. Students are encouraged to work in small groups on these questions to actively engage one another in the learning process. ${ }^{1-4}$ This interaction elicits more engagement from the students through student-student and student-instructor interaction, compared to a more traditional lecture format. ${ }^{4}$

Inherent to any PI lecture is the need to accept and immediately process feedback from the students during the lecture. The instructor may then adapt the lecture based on the feedback. This paper discusses an electronic apparatus that we have created, whose purpose is to acquire and immediately process in-class feedback from students during a PI-style lecture. We have been using this apparatus in our own PI efforts for 2 years and have found it to be very useful.

\section{ACQUIRING STUDENT FEEDBACK IN THE CLASSROOM}

From our experience, we have identified six areas that need to be addressed by an effective in-class feedback methodology. They are (1) speed, (2) instructor effort, (3) assimilation, (4) accuracy, (5) real-time and conciseness of results, and (6) rigor and accountability. Taking student feedback must be fast and not use much class time. Only a small additional fraction of the time allotted for the student work on the questions is allowable. The instructor must not need to put forth a considerable amount of attention or effort to measure student feedback, and the feedback method must be easily integrated into the usual class setting with minimal disruption to both the teacher and students. The instructor must have instant access to the student feedback results in a single, simple, and easy to read format. And most importantly, the feedback mechanism must be such that the students can see that their responses are being rigorously measured, and that it is in their best interest to try to be correct in their own response (which might include altering their outside class study habits).

We first summarize some common in-class feedback methods (some are taken from Ref. 1). The weaknesses of each are the motivation for this work.

An instructor can ask students to raise their hands in response to a question. We often wonder if students are raising their hands only when everyone else is and if everyone is participating. Also, in large (30+ student) classes, it can be difficult to count hands. This method also leaves little accountability for student answers.

Flash cards are a popular technique for implementing PI. ${ }^{1,4}$ Students are given (or make) a set of six or more cards labeled A-F, for example. Each student holds up a card in response to a (multiple choice) question. We find that when results are mixed, several visual passes through the classroom are required to tally all the responses. Also, distributing and collecting flash cards or asking students to remember to bring their flash cards can be problematic.

Mazur ${ }^{1}$ reports using Scantron forms that students use to "bubble in" their answers. Interpreting responses are obviously delayed because the forms must be scanned after class, so the instructor cannot adapt the lecture based on the responses. The scanning and record keeping creates a significant amount of extra work and logistics. This method also is wasteful of (paper) resources, particularly in large classes.

Handheld computers, calculators, and other devices, are available, ${ }^{5}$ whereby students input their responses into a wired or wireless device that subsequently transmits them to a centralized computer. These systems can be expensive and there can be problems with the logistics of distributing, collecting, and maintaining a class set of handheld devices. There are other possibilities involving computers, laptops, and (wireless) networks, ${ }^{6,7}$ which can be developed into feedback systems with strong accountability, but only after much equipment, developmental, and maintenance costs.

To address the problems with the above techniques, we have created an electronic, in-class, student feedback system. Although it requires some technical ability to assemble, the system is inexpensive to build (less than \$150), and uses many off the shelf components. The system is wholly operated by the instructor, gives immediate feedback results, is simple to set up, fairly inconspicuous, and requires only a single in-class computer. The system adds an element of rigor to the feedback process, and results are accurately compiled. Software control can add accountability to the students' answers by tying their responses to an identification number. The system has been found to be usable in classes of up to 50 students.

The system is a keyboard multiplexer. It is a custom electronic circuit that allows for the connection of up to 1-5 keyboards to a single computer. ${ }^{8}$ The computer is programmed to rapidly $(100 \mathrm{~Hz})$ poll the keyboards individually, accepting and categorizing input from each. Currently, the system only processes the letters $\mathrm{A}-\mathrm{G}$, as needed by answers to multiple choice questions. If more than one mul- 


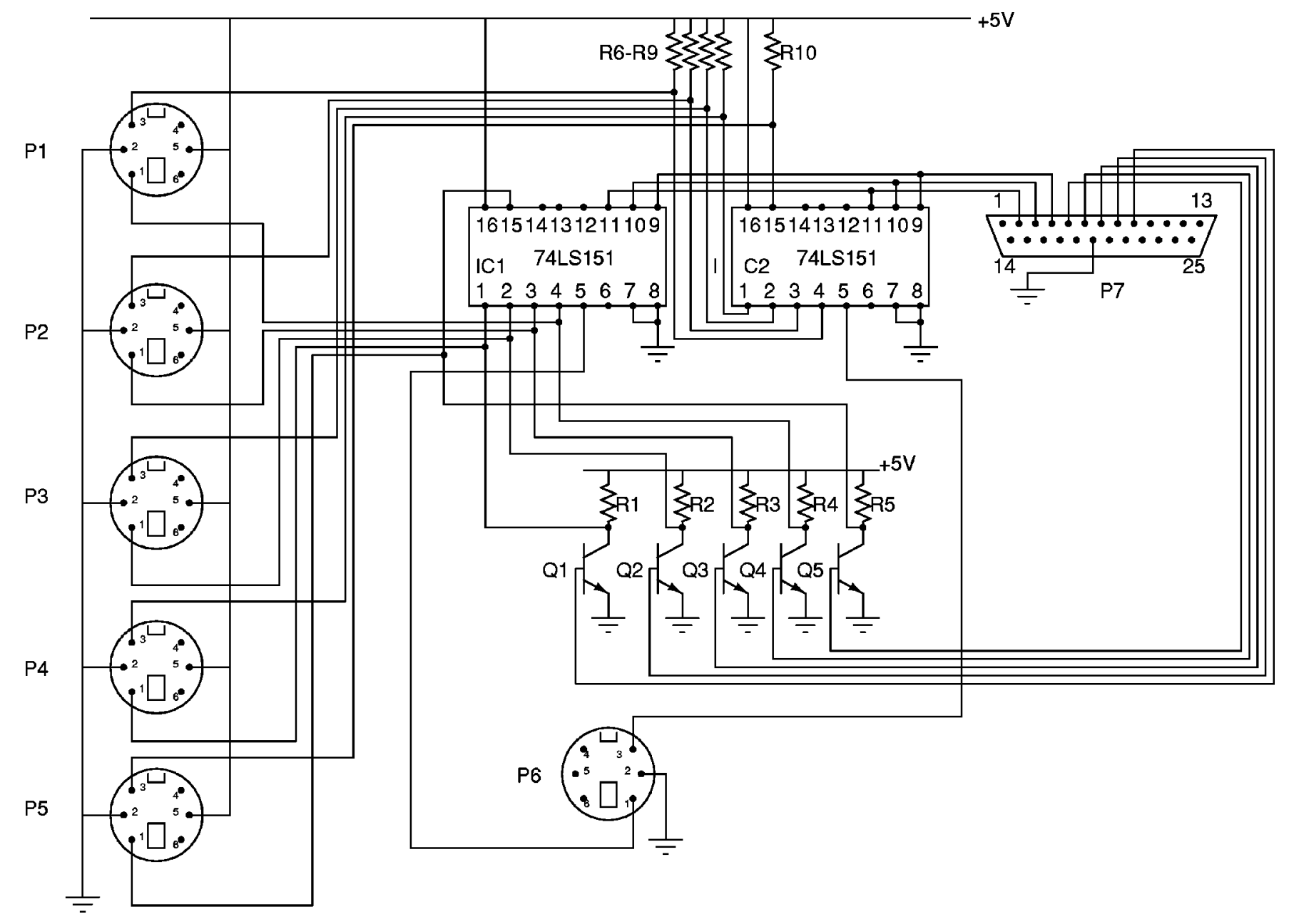

Fig. 1. The circuit that allows for up to five keyboards to be connected to and independently read by a single computer.

tiple choice question has been posed, the system accepts "answer strings," which is a single word derived from a concatenation of each answer with which the student wishes to respond. For example, if a student answers a for question 1 , $\mathrm{d}$ for question 2 , and $\mathrm{b}$ for question 3 , the answer string would be "adb." During or after the student input process, the instructor can view individual histograms on the screen, and see the distribution of answers for a given question.

Using the system in the context of an interactive lecture proceeds as follows. When the students are ready to input their responses, they go to the area where the feedback system has been set up (typically at the front of the classroom) to type in their answer or answer string. They are instructed to use any available keyboard, up to the five possible. Also, for group work, it can be requested that only one student per group input the answers representing the effort of the entire group. Note that this answer method is completely anonymous. Answer strings that students input to the system may be prefixed with a fixed-length identification number. In this case, the in-class behavior of the system remains the same, but internally, the identification number and answers are paired and then stored to disk for later access by the instructor. This method of accepting answers is anonymous only to the students, as they interact in the classroom, but each student is now accountable for their answers.

A concern mentioned earlier is with the speed of this system in acquiring the feedback. This (or any) feedback method must not unnecessarily consume valuable class time. When a question-activity is initiated, it is stressed that the students are to input their answers immediately upon arriving at their final answers, not when time is called on the activity itself. This procedure uses time efficiently and avoids lines of students waiting to input their answers. At any given time, we have found that between one and four students are inputting their answers, which also approximately corresponds to the number of students moving about the classroom. Even in a class of 50 students, we have yet to experience any significant in-class disruption, loss of time, or lines of students waiting to input their answers.

When all of the students have inputted their responses, the instructor can visually observe and interpret the distribution of answers. The computer display can be viewed only by the instructor or projected onto a screen (using a suitable computer projector) for the entire class to observe. The instructor can now adapt the lecture based on the results.

The required functionality of this feedback system is fiveto-one keyboard multiplexing. Because a personal computer only has a single keyboard input port, the output of up to five keyboards must be carefully coordinated and synchronized, so that only one keyboard transmits data into the computer at a time. The electronic circuit to perform this task is shown in Fig. 1. When complete, the circuit includes five female PS/ 2-style keyboard connectors and one male PS/2 keyboard connector. The keyboards that the students use are connected 
to the circuit via the five female connectors. These keyboards are off-the-shelf PC keyboards with PS/2 style connectors. Full sized keyboards may be used as can small 16-key numeric keypads,${ }^{10}$ for increased system portability. The PS/2 male connector on the circuit is plugged into the normal keyboard port of the computer, so that it can accept input from the keyboards via this circuit. The circuit is externally powered by a $5 \mathrm{~V}, 1.5 \mathrm{~A}$ power supply, and interfaces to the computer via the computer's parallel (printer) port. Technical notes about the circuit are given in Appendix A.

Building the apparatus requires some electronic circuit knowledge and assembly skills, but not beyond an upper division physics course on electronics, or what a typical physics department technician can provide. The total cost of all the components required to build the circuit is $\approx \$ 50$. The circuit should take approximately one week to construct. The keyboards can increase the cost of the project, depending on the type and number used. It is likely that surplus keyboards from older computer equipment can be obtained for a nominal cost.

\section{CONCLUSIONS}

We have discussed an electronic, in-class feedback system that satisfies the criteria we desire in an in-class feedback system (see Sec. II). A required element of this feedback system is that students must get out of their seats and approach the system to input their answers. From our experience in using this system, this procedure does not cause any undue class disruption or inconvenience. In fact, we find this physical activity comes as a welcome break during a lecture, rejuvenating the students' energy and attention. A copy of the software discussed in this paper is available on EPAPS. ${ }^{11}$

\section{APPENDIX A: CIRCUIT}

Our software ${ }^{11}$ for an Intel-based personal computer selects a keyboard from which the computer would like to accept input. Only keyboard is allowed to send data to the computer at any one time through the computer's standard keyboard interface. The electrical components include two 74LS151 8-input multiplexers (IC1 and IC2), five 2N2222 (or equivalent) NPN transistors (Q1-Q5), and ten $1 \mathrm{~K}$ resistors (R1-R10) (see Fig. 1).

Connectors P1-P5 are female 6-pin mini-DIN connectors (Jameco 9 119474). We can plug into these connectors five standard keyboards that have 6-pin mini PS/2 type connectors. ${ }^{12}$ Connector P6 is a male 6-pin din connector (the male version of Jameco 119474). This connector looks like the one at the end of the cable attached to a keyboard with a 6-pin mini male PS/2 type connector. The circuit is connected to the keyboard input on the computer via this connector and a standard 6-pin male/female keyboard extension cable. Connector P7 in Fig. 1 is a male 25-pin IEEE 1284-A D sub connector. This connector mates with the parallel printer port connector on the back of a personal computer. The circuit is connected to a parallel port via this connector and a standard 25-pin (male/female) 25-pin cable. The final connector is a power connector that will accept a standard $+5 \mathrm{~V} \mathrm{DC}, 1.5$ A wall transformer such as Jameco \#161664. ${ }^{9}$

The computer selects which keyboard it will accept input from by simultaneously writing TTL logic levels from the parallel port to pins 11,10 , and 9 of both 74LS151 multi- plexers. Both 74LS151s are always set to the same address at the same time via direct electrical connections between the address pins on both 74LS151s.

Because the keyboard uses two lines in communicating with the computer (a "clock" line and a "data" line), both must be simultaneously multiplexed by this circuit. In the circuit (Fig. 1), the leftmost 74LS151 multiplexes the clock lines from the keyboards, and the rightmost 74LS151 multiplexes the data lines. Clock lines are connected to the pull-up resistors on the NPN transistors so the software may allow or inhibit a keyboard from sending any data to the system. Allowing or inhibiting a keyboard is controlled by the on/off state of each transistor, which is controlled through software via transistor base connections to the parallel port. A keyboard must be inhibited from sending input, for example, when another keyboard has been selected for input.

\section{APPENDIX B: COMPATIBILITY WITH NEWER OPERATING SYSTEMS}

As described in Appendix A, the keyboard input is controlled and coordinated using the parallel port. This port is rapidly being replaced by the Universal Serial Bus (USB). Furthermore, the most recent versions of the Windows operating system, such as NT, 2000, and XP, do not support using the parallel port in the manner required by this system.

To make this system available to those using these new operating systems, the parallel port control portion of this system can be replaced with the ActiveWire USB development device. ${ }^{13}$ Only small changes to the circuit are needed to integrate this device. First IO0, IO1, and IO2 of the ActiveWire board are wired to pins 11,10 , and 9 on the 74LS151 pairs. These lines become the new keyboard selection method. Second, ActiveWire lines IO3, IO4, IO5, IO6, and IO7 are wired to the bases of transistors Q1, Q2, Q3, Q4, and $\mathrm{Q} 5$, respectively. These lines become the new keyboard allow/inhibit controls. A USB version of the system software is available ${ }^{11}$ to control the system via the ActiveWire board. We have fully integrated the ActiveWire board into our design and have found it to perform flawlessly.

${ }^{1}$ Eric Mazur, Peer Instruction: A User's Manual (Prentice-Hall, Upper Saddle River, NJ, 1997).

${ }^{2}$ C. H. Crouch and E. Mazur, "Peer instruction: Ten years of experience and results," Am. J. Phys. 69, 970-977 (2001).

${ }^{3}$ A. P. Fagen, C. H. Crouch, and E. Mazur, "Peer instruction: Results from a range of classrooms," Phys. Teach. 40, 206-209 (2002).

${ }^{4}$ D. E. Meltzer and K. Manivannan, "Transforming the lecture-hall environment: The fully interactive physics lecture," Am. J. Phys. 70, 639-654 (2002) and references therein.

${ }^{5}$ Other handheld devices can be found at Classtalk, 〈http:// education.ti.com); Classroom Performance System, 〈http:// www.einstruction.com $\rangle$, Personal Response System, 〈http:// www.educue.com $>$

${ }^{6}$ IBM Corp. advertisement, "T.H.E. Journal: Technological Horizons in Education," 29, 11 (2002).

7“CSU Quiz," California State University, Exchanges Journal, Winter/ Spring 2001 (online only). 〈http://www.exchangesjournal.org

${ }^{8}$ More than one device is needed to avoid a logjam of students waiting to input their answers. We have found that one input device per 10 students is adequate.

${ }^{9}$ Jameco Electronics, 1355 Shoreway Blvd., Belmont, CA 94002-4100, $\langle$ http://www.jameco.com〉

${ }^{10} \mathrm{See}$, for example, the Micro Innovations KP17B Numeric Keypad at $\langle$ http://www.amazon.com〉. Most small portable keypads like this contain 
only numeric keys. In order to process letters for the answers to multiple choice questions, each numeric key is outfitted with a single letter of the alphabet in the form of a $1 / 4^{\prime \prime}$ vinyl sticker. Our software can convert numeric input from the keypad to a letter for feedback processing.

${ }^{11}$ See EPAPS Document No. E-AJPIAS-71-002309 for one of two software programs for Windows-based computers. The first runs the circuit using the parallel port. The other runs the circuit from the USB port. A direct link to this document may be found in the online article's HTML reference section. The document may also be reached via the EPAPS homepage (http://www.aip.org/pubservs/epaps.html) or from ftp.aip.org in the directory/epaps. See the EPAPS homepage for more information.

${ }^{12}$ Although the 74LS151 can multiplex eight digital lines, only five keyboards are accepted by this system due to a maximum of eight output lines on the PC's parallel port. Five of the output lines are used to allow or inhibit a keyboard's input, and the remaining three are used as address lines to the multiplexers.

${ }^{13}$ T. Z. Fullem and C. D. Spencer, "A universal serial bus interface for electronics projects and instruments,” Am. J. Phys. 70, 972-974 (2002). 